\title{
RESEARCH
}

Open Access

\section{Identification of repurposable cytoprotective drugs in vanishing white matter disease patient-derived cells}

Neville Ng ${ }^{1,2}$, Mauricio Castro Cabral-da-Silva ${ }^{1,2}$, Simon Maksour ${ }^{1,3}$, Tracey Berg $^{1,2}$, Martin Engel ${ }^{1,2}$, Dina M. Silva ${ }^{1,2}$, Dzung Do-Ha, $a^{1,2}$, Jeremy S. Lum ${ }^{1,2}$, Sonia Sanz Muñoz ${ }^{1,2}$, Nadia Suarez-Bosche ${ }^{1,2}$, Claire H. Stevens ${ }^{1,2}$ and Lezanne Ooi ${ }^{1,2^{*}}$ (iD

\begin{abstract}
Background: Vanishing white matter disease (VWMD) is a rare disease caused by mutations of the guanine exchange factor eIF2B. WWMD typically presents with juvenile onset, and there are few treatments for the disease. Recent progress in the field has established mitochondrial dysfunction and endoplasmic reticulum (ER) stress to be strongly implicated in observed glial cell pathology. Drug repurposing offers a rapid approach toward translation of therapeutics using already-licensed drugs.

Objective: The aim of this study was to use fibroblasts and induced pluripotent stem cell (iPSC)-derived astrocytes from patients bearing the EIF2B5 $5^{R 113 \mathrm{H} / \mathrm{A} 403 \mathrm{~V}}$ or EIF2B2 $2^{\mathrm{G} 200 V / E 213 G}$ WWMD mutations to identify potential repurposable FDA-approved drugs based on in vitro assays.

Methods: Cell viability in the presence or absence of stress was assessed by resazurin reduction activity and assays for mitochondrial membrane potential and oxidative stress by tetramethylrhodamine and dichlorofluorescein-based assays, respectively. Integrated stress response markers, including elF2a phosphorylation, GADD34 and CHOP were quantified by fluorescent western blot.
\end{abstract}

Results: Dysregulated GADD34 and CHOP were identified in patient fibroblasts and iPSC-derived astrocytes under induced stress conditions. A drug screen from a 2400 FDA-approved drug library with EIF2B5 $5^{R 113 H / A 403 V}$ WWD patient fibroblasts identified 113 anti-inflammatory drugs as a major class of hits with cytoprotective effects. A panel of candidate drugs, including berberine, deflazacort, ursodiol, zileuton, guanabenz and Anavex 2-73, and preclinical ISRIB, increased cell survival of EIF2B5 $5^{\mathrm{R} 113 \mathrm{H} / \mathrm{A} 403 \mathrm{~V}}$ or EIF2B2 ${ }^{\mathrm{G} 200 \mathrm{~V} / \mathrm{E} 213 \mathrm{G}}$ WWMD astrocytes, and were further investigated for their effect on the integrated stress response and mitochondrial stress. Ursodiol demonstrated capacity to ameliorate oxidative stress and loss of mitochondrial membrane potential in VWMD patient iPSC-derived astrocytes in the presence or absence of stress conditions.

(Continued on next page)

\footnotetext{
* Correspondence: lezanne@uow.edu.au

${ }^{1}$ Illawarra Health and Medical Research Institute, Northfields Avenue,

Wollongong, 2522 New South Wales, Australia

${ }^{2}$ School of Chemistry and Molecular Bioscience and Molecular Horizons,

University of Wollongong, Northfields Avenue, Wollongong, 2522 New South

Wales, Australia

Full list of author information is available at the end of the article
}

(C) The Author(s). 2020 Open Access This article is licensed under a Creative Commons Attribution 4.0 International License, which permits use, sharing, adaptation, distribution and reproduction in any medium or format, as long as you give appropriate credit to the original author(s) and the source, provide a link to the Creative Commons licence, and indicate if changes were made. The images or other third party material in this article are included in the article's Creative Commons licence, unless indicated otherwise in a credit line to the material. If material is not included in the article's Creative Commons licence and your intended use is not permitted by statutory regulation or exceeds the permitted use, you will need to obtain permission directly from the copyright holder. To view a copy of this licence, visit http://creativecommons.org/licenses/by/4.0/. 
(Continued from previous page)

Conclusion: Patient-derived cells can be used to identify cellular phenotypes and for large-scale drug screening. Anti-inflammatory compounds, such as berberine, deflazacort, ursodiol and zileuton are potentially repurposable drug candidates for WWMD that should be further investigated for translation in vivo.

Keywords: Vanishing white matter disease, Leukodystrophy, Cell stress, Drug repurposing, Induced pluripotent stem cells, Astrocytes

\section{Background}

Vanishing white matter disease (VWMD) is a rare, autosomal recessive disease, caused by mutations in the genes EIF2B1-5, encoding the eukaryotic initiation factor eIF2B [1]. The eIF2B protein is a guanine nucleotide exchange factor that is involved in the integrated stress response (ISR). VWMD mutations can cause alterations in the activity of the wild-type eIF2B protein and the disease has a variable onset, progression and severity, which can be exacerbated by environmental stress factors [2]. Although the disease course varies widely, earlier disease onset is associated with a more rapid progression and patients developing symptoms as young children typically survive few years beyond diagnosis [3].

The eIF2B proteins control mRNA translation, converting the inactive eIF2-GDP to the active eIF2-GTP form. Activation of the cytoprotective ISR leads to phosphorylation of eIF $2 \alpha$ (p-eIF2 $\alpha$ ), which binds to eIF2B to mediate translational repression, and upregulation of stress-induced genes. These genes include PPP1R15A that encodes growth arrest and DNA damage-inducible protein (GADD34). GADD34 facilitates dephosphorylation of p-eIF $2 \alpha$ to promote recovery from stress and resumption of normal protein translation in a negative feedback loop, and transcription factors ATF4 and CHOP [4]. Mutations in eIF2B can lead to delayed translation of stress-induced genes and dysregulated ISR [5]. Although eIF2B is ubiquitously expressed and plays a role in multiple cell types, the disease manifests most significantly in the loss of white matter of the brain [6].

VWMD mouse models and induced pluripotent stem cell (iPSC) models [7, 8] have identified a central role for dysfunctional astrocytes in the development of VWMD, with evidence for astrocytic apoptosis [8] and an inability to promote oligodendrocyte maturation [6]. A key driver of cellular pathogenesis in VWMD involves the ISR, with alterations in responses to endoplasmic reticulum stress and oxidative stress [9, 10]. Mitochondrial dysfunction and increased accumulation of reactive oxygen species have been identified in VWMD murine fibroblasts, astrocytes and oligodendrocyte precursor cells [11, 12]. Currently there are no approved treatments for VWMD, hence the aim of this research was to identify candidates from an FDA-approved drug library that could protect VWMD patient cells against cellular stress. This pre- clinical research can be used to inform future in vivo studies. For phenotypically diverse diseases, such as VWMD, patient-derived cells provide a useful model of disease mutations that can aid translational pathways.

\section{Materials and methods}

\section{Cell culture}

All experimental protocols were approved by the University of Wollongong Human Research Ethics Committee (HE17/522). Primary human dermal fibroblasts were collected from two VWMD patients that were diagnosed in early childhood or their control family members. The VWMD1 iPSC line was generated from a patient bearing mutations in EIF2B $5^{\mathrm{R} 113 \mathrm{H} / \mathrm{A} 403 \mathrm{~V}}$, whilst the VWMD6 iPSC line was generated from a patient bearing mutations in $E I F 2 B 2^{G 200 V / E 213 G}$. The VWMD1 control line is a healthy control (no disease mutations, EIF2B $5^{\mathrm{R} 113 / \mathrm{A} 403}$ ), whilst the VWMD6 control bears one VWMD allele and one wildtype allele (non-disease carrier, EIF2B2 $2^{\mathrm{G} 200 \mathrm{~V} / \mathrm{E} 213}$ ). Cells were maintained in DMEM/F12 (Gibco, Ireland, 21331020), 10\% FBS (Bovogen Biologicals, Australia, SFBS-AU), 2 mM L-glutamine (Gibco, Ireland, 25030081) and $1 \%$ penicillin/streptomycin (Gibco, 15140122). Fibroblasts were reprogrammed with the StemRNA (ReproCell, Beltsville, Maryland, 00-0076) non-integrating method, as previously described $[13,14]$. The mRNA-based reprogramming of patient fibroblasts into iPSCs, pluripotency marker immunofluorescence and RT-qPCR, karyotyping and mutation genotype characterisation are described in Supplementary Information (Figure S1). The iPSCs were maintained in mTESR1 (Stem Cell Technologies, Australia, 85850) and astrocytes in Astrocyte Growth Supplement Medium (AGS; ScienCell Research Laboratories, San Diego, California, USA, 1852). All cells were maintained in humidified incubators at $37^{\circ} \mathrm{C}$ supplemented with $5 \% \mathrm{CO}_{2}$ for fibroblasts or hypoxic $3 \% \mathrm{O}_{2}$ conditions for iPSCs and astrocytes. Neural inductions were carried out as previously described [15]. Astrocyte differentiations were performed in ciliary neurotrophic factor (CNTF), epidermal growth factor (EGF) and basic fibroblast growth factor (FGF-2)-based medium and transitioned to AGS, prior to characterisation by immunofluorescence and inflammatory activation, to confirm the production of functional astrocytes from iPSCs. Details of neural inductions and astrocyte characterisations are in Supplementary 
Information (Figure S2). Genotyping and characterisation RT-qPCR primers are listed in Table S1.

\section{Cell stress and viability assays}

Fibroblasts or astrocytes were seeded in 96 well plates (5000 cells/well) and incubated with a range of concentrations of hydrogen peroxide $\left(\mathrm{H}_{2} \mathrm{O}_{2} ; 0-2 \mathrm{mM}\right.$, SigmaAldrich, 16911), MG132 (0-20 $\mu \mathrm{M}$; Focus Bioscience, HY-13259) or thapsigargin (0-20 $\mu \mathrm{M}$; Abcam, Australia, ab120286) overnight. Viability was assessed via resazurin reduction assay (STEMCELL Technologies, 75005), in which cells received $15 \mu \mathrm{M}$ resazurin for $1 \mathrm{~h}$ prior to measurements by fluorescence plate spectroscopy (excitation 544/emission $590 \mathrm{~nm}$ ). For coincubation assays, cells were similarly prepared and incubated with candidate drugs and, based on the $\mathrm{IC}_{60}$ for each cell type, MG132 at either $2.5 \mu \mathrm{M}$ (fibroblasts) or $0.1 \mu \mathrm{M}$ (astrocytes) for $48 \mathrm{~h}$. The coincubation drug screen was carried out in VWMD1 patient fibroblasts with the MicroSource Spectrum FDA collection (Compounds Australia), a library of 2400 FDA-approved drugs. Cell seeding and drug reagent preparation were performed by a robotic liquid handler (Hamilton Microlab STAR, Reno, Nevada). First-pass screening of the drug library was carried out at $20 \mu \mathrm{M}$; drug candidates selected for further testing underwent dose-response curves to identify appropriate concentrations. Single drug concentrations were selected based on protective efficacy in dose-response coincubation assays and used in downstream assays at concentrations of: Anavex 2-73 (AVex73, $5 \mu \mathrm{M}$; Focus Bioscience, Australia, HY-101864), berberine $(1.25 \mu \mathrm{M}$; Sigma-Aldrich, Australia, 14050), guanabenz $(5 \mu \mathrm{M}$; Focus Bioscience, HY-B0566), ISRIB $(1.25 \mu \mathrm{M}$; Focus Bioscience, HY-12495), deflazacort ( $5 \mu \mathrm{M}$; Sigma-Aldrich, SML0123), ursodiol $(40 \mu \mathrm{M}$; Sigma-Aldrich, PHR1579) and zileuton $(1.25 \mu \mathrm{M}$; Focus Bioscience, HY-14164). A DMSO (Sigma-Aldrich, D4540) vehicle (solvent) concentration of $0.2 \%$ was included in all experiments.

\section{Integrated stress response protein quantification}

Fibroblasts or astrocytes were seeded in 6 well plates (200, 000 cells/well) and incubated with MG132 for 0, 24 or 48 $\mathrm{h}$, or coincubated with candidate compound and MG132 overnight. Cultures were lysed in RIPA buffer $(50 \mathrm{mM}$ Tris, $\mathrm{pH} 8.0,150 \mathrm{mM} \mathrm{NaCl}, 1 \%$ Triton X-100, $0.5 \%$ sodium deoxycholate, $0.1 \%$ SDS and protease inhibitors cOmplete Protease Inhibitor (Roche, 4693116001) and phosphatase inhibitors PhosSTOP EASY (Roche, 4906837001)) before sonication and quantification by a Pierce BCA Protein Assay Kit (Thermo Fisher Scientific, 23225). Protein lysates were heated for $5 \mathrm{~min}$ at $95^{\circ} \mathrm{C}$ in loading buffer (2\% SDS, 10\% 2-mercaptoethanol, 10\% glycerol, $0.001 \%$ bromophenol blue, $0.0625 \mathrm{M}$ Tris- $\mathrm{HCl}, \mathrm{pH}$
6.8). Denatured protein lysates $(5 \mu \mathrm{g})$ were separated on 4-20\% Criterion TGX Precast Gels (Bio-Rad, Australia, 5678095) and transferred to Immobilon-FL PVDF membrane $(0.45 \mu \mathrm{m}$ pore; Merck, Darmstadt, Germany, IPFL00010). Membranes were blocked with $0.5 \%$ casein (Bio-Rad, 1610782) for $1 \mathrm{~h}$ (RT), primary antibody at 1 : 1000 dilution for $16 \mathrm{~h}\left(4^{\circ} \mathrm{C}\right)$, and secondary antibody at 1 : 5000 dilution for $1 \mathrm{~h}\left(4^{\circ} \mathrm{C}\right)$, facilitated by an automated western blot processor (Cytoskeleton GOBlot, Denver, Colorado, USA). Antibodies utilised included anti-eIF2 $\alpha$ (Abcam, ab5369), anti-eIF2 $\alpha$-phopsho-S51 (Abcam, ab32157), anti-DDIT3 (DNA damage inducible transcript 3, also known as C/EBP homologous protein (CHOP; Abcam, ab179823), anti-GADD34 (Sigma-Aldrich, SAB2701237), donkey anti-mouse Alexa Fluor Plus 488 (Thermo Fisher Scientific A32766) and donkey antirabbit Alexa Fluor Plus 647 (Thermo Fisher Scientific A32795). Blots were imaged with a Bio-Rad ChemiDoc MP. Band intensities were normalised to stain-free total protein. Relative eIF2 $\alpha$ phosphorylation was normalised to total eIF2 $\alpha$. Dynamic range of signal was confirmed by linear signal to loading ratio (Figure S3).

\section{Oxidative stress, mitochondrial membrane potential and membrane integrity microscopy assays}

Astrocytes were seeded in 96 well plates $(10,000$ cells per well) and coincubated overnight with MG132, as described above, and candidate drugs, or the positive controls for oxidative stress: $\mathrm{H}_{2} \mathrm{O}_{2}(100 \mu \mathrm{M})$, or the proton uncoupler carbonyl cyanide-4-phenylhydrazone (FCCP; $10 \mu \mathrm{M}$; Focus Bioscience, HY-100410). Cells were loaded with $20 \mu \mathrm{M}$ H2DCFDA (DCF; Thermo Fisher Scientific, D399) or $0.5 \mu \mathrm{M}$ tetramethylrhodamine ethyl ester (TMRE; Thermo Fisher Scientific, T669) and $10 \mu \mathrm{M}$ Hoechst 33342 (Sigma-Aldrich, B2261) for $0.5 \mathrm{~h}$ before replacement of medium with phenol red-free DMEM/F12 medium (Gibco, 21,041,025) and further incubation for $1 \mathrm{~h}$. Relative fold change in DCF signal was acquired by fluorescence spectroscopy and normalised to cell density quantified by $0.004 \%$ sulforhodamine B (Sigma-Aldrich, 230162) solubilised in $10 \mathrm{mM}$ Trizma (Sigma-Aldrich, T1503) after fixation in 4\% trichloroacetic acid (Sigma-Aldrich, T9159) [16]. TMRE images were acquired by confocal microscopy, TMRE (excitation 522/emission 590-620 nm) and analysed with ImageJ to determine fluorescence normalised to cell density.

\section{Statistical analyses}

Data are presented as the mean and standard error of the mean of at least 3 independent experiments; statistical significance was assessed by two-way analysis of variance followed by Holm-Sidak posthoc test for multiple comparisons unless otherwise stated. 
Significance was accepted where $p<0.05$. Charts and statistical analyses were prepared in Prism GraphPad 8.0.

\section{Results \\ VWMD patient fibroblasts and astrocytes exhibit dysregulated ISR marker expression}

Mutations in eIF2B suppress both global and stressinduced protein translation, in response to ER stress [5, 17]. Given that eIF2B is ubiquitously expressed, we examined patient fibroblasts and iPSC-derived astrocytes for evidence of a dysfunctional ISR. Fibroblasts were reprogrammed into iPSCs from two VWMD patients, along with gender-matched relatives as non-disease controls (Figure S1). A previous study identified that white matter-derived astrocytes, generated using a ciliary neurotrophic factor (CNTF)-based differentiation protocol, showed a more vulnerable phenotype to stress, compared to grey matter astrocytes generated using fetal bovine serum [7]. Consequently, we generated astrocytes from iPSCs using a CNTF-based method (Figure S2).

Responses to oxidative and endoplasmic reticulum (ER) stresses are all proposed to be affected in VWMD cell and animal-based models [17]. Studies employing cells bearing different VWMD mutations have shown variability in the ISR [5], and primary astrocytes from mice bearing a homozygous R191H mutation in eIF2Be did not exhibit the ISR in vitro [18]. Thus, first we established stress conditions that could generate an exacerbated cellular phenotype in the VWMD patient cells compared to controls. We evaluated the dose-dependent effect of $\mathrm{H}_{2} \mathrm{O}_{2}$, as a mediator of oxidative stress, thapsigargin, as a mediator of ER stress, and MG132. MG132 is commonly utilised as a proteasomal inhibitor that also induces ER and oxidative stress via the unfolded protein response, all of which trigger the ISR [19, 20]. There was a small but significant reduction in cell survival for VWMD fibroblasts and iPSC-derived astrocytes, compared to non-disease controls, under all three stressors (Figure S4A).

Disrupted ISR homeostasis can be assessed by measuring the expression levels of ISR-relevant markers. The control of eIF $2 \alpha$ phosphorylation or dephosphorylation acts as a pivotal mechanism that regulates global protein synthesis in response to cell stress [21]; GADD34 dephosphorylates eIF2 $\alpha$ [22] while CHOP is activated by the ISR to promote apoptosis [23]. The expression levels of these ISR-relevant proteins, p-eIF2 $\alpha$ (normalised to eIF2 $\alpha$ ), GADD34, and CHOP, were compared in VWMD and control fibroblasts, and iPSC-derived astrocytes, under MG132 stress (Fig. 1; antibody characterisation and representative blots shown in Figure S3-4). VWMD lymphoblasts have previously been reported to show reduced GADD34 expression and increased levels of p-eIF2 $\alpha$, following thapsigargin-induced acute ER stress [5]. Under MG132-induced stress, VWMD fibroblasts showed reduced upregulation of eIF $2 \alpha$ phosphorylation and reduced GADD34 expression, and increases in CHOP expression, compared to controls (Fig. 1a-c). MG132-stressed VWMD astrocytes also showed reduced upregulation of eIF2 $\alpha$ phosphorylation and increased CHOP, compared to controls (Fig. 1d-f). VWMD astrocytes exhibited increased GADD34 (Fig. 1e), consistent with glia in animal model studies [17] and the hypophosphorylation of eIF2 observed in white matter patient tissue [24].

\section{Cytoprotective drug screen in VWMD patient cultures}

Based on the established capacity of MG132 to induce oxidative stress, and exacerbate ISR disease phenotypes, we performed a first-pass drug screen for candidates able to protect against the effect of low dose MG132 in VWMD1 EIF2B $5^{\mathrm{R} 113 \mathrm{H} / \mathrm{A} 403 \mathrm{~V}}$ patient fibroblasts, with cell viability assessed by resazurin reduction activity (Fig. 2).

Following the initial drug screen, a panel of 20 compounds (Table S2) was selected for downstream evaluation in VWMD1 EIF2B5 $113 \mathrm{H} / \mathrm{A} 403 \mathrm{~V}$ fibroblasts and in both VWMD1 EIF2B5 $5^{\mathrm{R} 113 \mathrm{H} / \mathrm{A} 403 \mathrm{~V}}$ and VWMD6 $E I F 2 B 2^{\mathrm{G} 200 \mathrm{~V} / \mathrm{E} 213 \mathrm{G}}$ patient-derived astrocyte cultures. The compounds selected for downstream assays were chosen based on their potential for clinical translation, including considerations of bioavailability, route of administration and potential side effects. The drug panel included 14 protective compounds from the screen (> $1.5 \times$ standard deviation of MG132-stressed controls), and a further six compounds with relevant modes of action for VWMD. The sigma-1 receptor pathway was recently identified in a drug screen to protect against mitochondrial dysfunction in a murine $E I F 2 B 5^{\mathrm{R} 132 \mathrm{H} /}$ $\mathrm{R} 132 \mathrm{H}$ model [11]. Nominated drugs on the basis of relevant mode of action to VWMD, included guanabenz [25], ISRIB [26], sigma-1 receptor agonists, AVex-73 and amitriptyline [11], as well as the central nervous system (CNS) cytoprotective compounds tauroursodeoxycholic acid and alkaloid berberine [27]. Overall, the panel of compounds for further evaluation included glucocorticosteroids, bile acids, iron chelators, antioxidants, ISR modulators and sigma-1 receptor agonists. All candidates elicited cytoprotective effects against MG132induced stress at varying concentrations in VWMD1 fibroblasts, with the exception of ISRIB. Ursodiol and its taurine derivative, tauroursodeoxycholic acid, showed similar cytoprotective efficacies. Anti-inflammatories were amongst the largest category of compounds that improved cell viability under proteasomal stress, with a high proportion of these being glucocorticosteroids. The recent demonstration of mitochondrial dysfunction and 
A

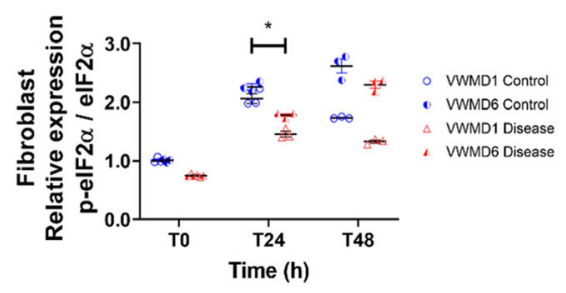

B

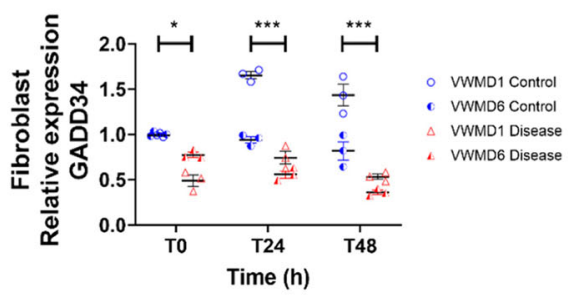

C

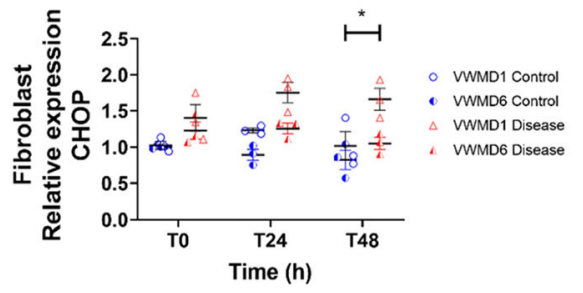

D

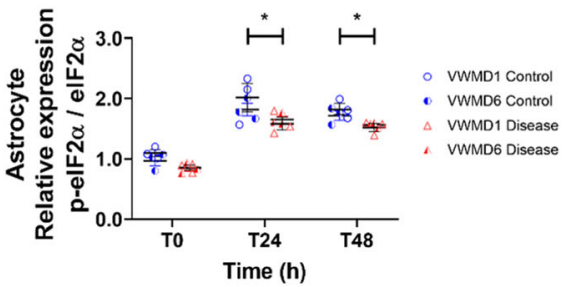

$\mathbf{E}$

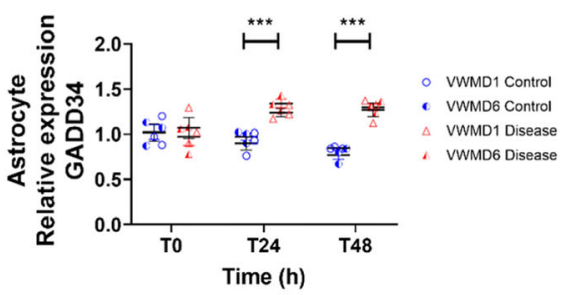

$\mathbf{F}$

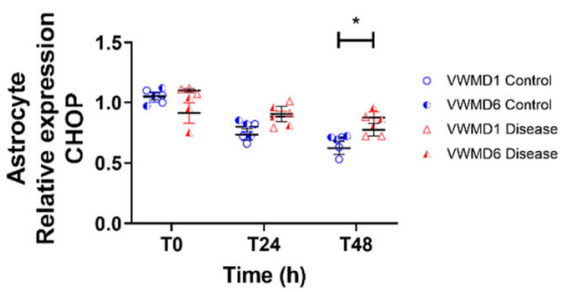

Fig. 1 ISR marker protein expression is affected in WWMD fibroblasts and astrocytes. a-f Effect of MG132 stress on protein expression of the ISR markers, p-elF2a (normalised to elF2a), GADD34 or CHOP, at 0, 24 or $48 \mathrm{~h}$ incubation period. Protein levels were quantified by western blot,

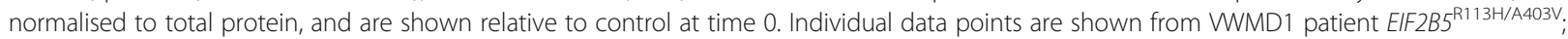
WWMD6 patient EIF2B2 ${ }^{\mathrm{G} 200 \mathrm{~V} / \mathrm{E} 213 \mathrm{G}}$ or their non-disease controls WWMD1 Control EIF2B5 ${ }^{\mathrm{R} 113 / \mathrm{A} 403}$; WWMD6 Control EIF2B2 ${ }^{\mathrm{G} 200 \mathrm{~V} / \mathrm{E} 213}$, with mean \pm SEM, $n=3$. Significant differences between pooled disease and control groups were identified by two-way ANOVA followed by Holm-Sidak post hoc test, ${ }^{*} p<0.05,{ }^{* *} p<0.01,{ }^{* * *} p<0.001$. Representative blots shown in (Figure S4B)

inefficient respiration in murine models of VWMD [11] has expanded the search for possible therapeutics to include mitochondrial protective compounds and antioxidants. The sigma-1 receptor is a chaperone protein in ER membranes that governs a range of cellular processes, including calcium homeostasis and reactive oxygen species accumulation [28]. However, in our study, the cytoprotective effect of the sigma-1 receptor agonists, AVex-73 and amitriptyline, was limited at higher concentrations ( $\geq 5 \mu \mathrm{M}$; Fig. 2, S5). Drugs assessed in iPSC-derived astrocytes from VWMD1 EIF2B $5^{\mathrm{R} 113-}$ H/A403V and VWMD6 EIF2B2 ${ }^{\mathrm{G} 200 \mathrm{~V} / \mathrm{E} 213 \mathrm{G}}$ yielded comparable results (Figure S5). The majority of the drugs that were protective in VWMD1 fibroblasts were also protective in VWMD1 and VMWD6 astrocytes, with the exception of amitriptyline, curcumin and budesonide (Fig. 2, S5).

Further studies were conducted to gain insight into the mode of action of drugs with therapeutic potential for VWMD (Fig. 2, S5), including AVex-73, berberine, deflazacort, guanabenz, ISRIB, ursodiol and zileuton. Assays to investigate drug mechanisms were performed with VWMD1 EIF2B5 $5^{\text {R113H/A403V }}$ patient astrocytes, given that the majority of VWMD-causing mutations affect the eIF2B $\varepsilon$ subunit [3].

\section{Effect of drug candidates on cell death in VWMD iPSC- astrocytes}

To determine whether candidate drugs reduced the proportion of bulk cell death, the membrane permeabilisation of treated astrocyte cultures was evaluated. In MG132-stressed VWMD1 astrocytes there were no significant changes in the percentage of membranepermeabilised cells, with the exception of ISRIB increasing the proportion of membrane-permeabilised cells (Figure S6). To test whether the drugs caused a reduction in mitochondrial apoptosis, the $B A X: B C L 2$ ratio was evaluated. The ratio of $B A X: B C L 2$ is used to assess the levels of Bax (proapoptotic) and Bcl-2 (antiapoptotic) expression. There was no detectable change in $B A X: B C L 2$ 


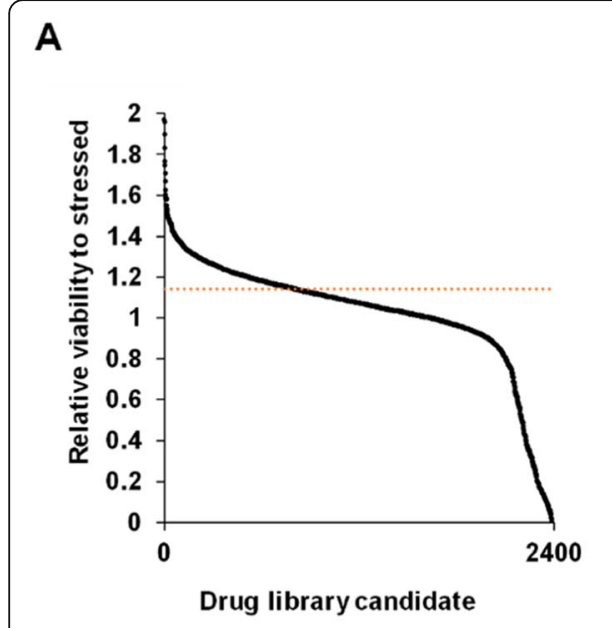

B

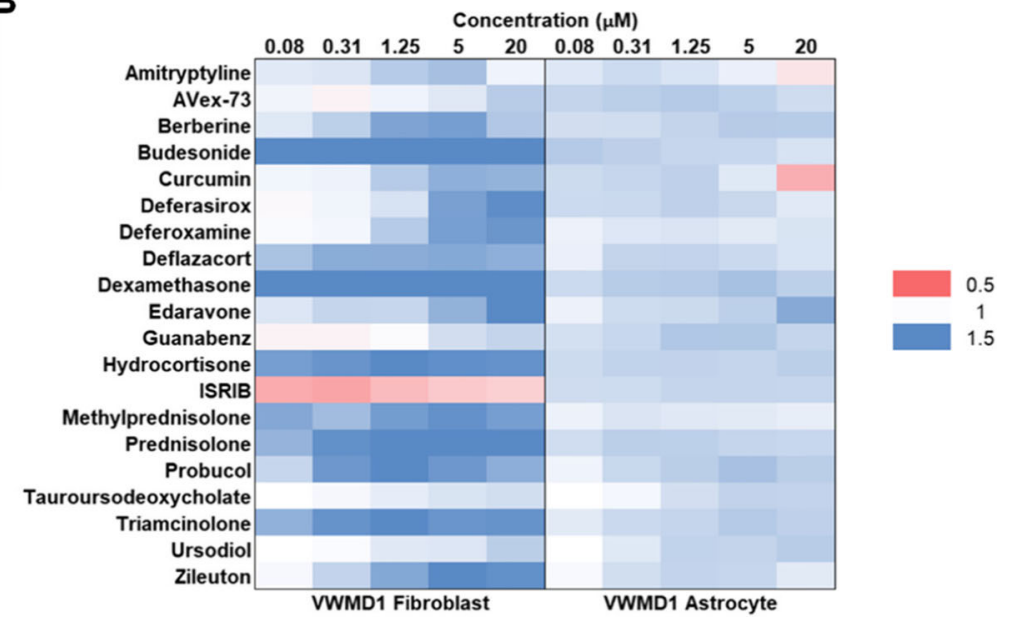

Fig. 2 Drug screen of cytoprotective candidates. a WWMD1 EIF2BR R113H/A403V disease fibroblasts were coincubated with MG132 and each of 2400 drugs from an FDA-approved drug library. Cell viability was measured and normalised to cell viability with MG132 stressor in the absence of drug. Drugs were scored based on increase in cell viability with threshold set to $>1.5 \times$ standard deviation $(n=3)$. Cytoprotection ranged from 2 -fold increase in viability to $0(100 \%$ cell death for cytotoxic drugs). Horizontal dotted red line indicates $1.5 \times$ standard deviation of MG132 stressed controls. b Hit candidates were taken forward to assess their dose-response effect on cell viability of WWMD1 EIF2B5 ${ }^{\mathrm{R} 113 \mathrm{H} / \mathrm{A} 403 \mathrm{~V}}$ fibroblasts and iPSC-derived astrocytes, relative to MG132 stress $(n=5-6)$. Heat map represents cytoprotection (blue) versus cytotoxicity (red) of individual drugs

ratio caused by any of the drug candidates, with the exception of an increase in $B A X: B C L 2$ caused by ISRIB (Figure S6).

\section{Effect of candidate drugs on ISR-relevant proteins p- elF2a, GADD34 and CHOP}

Candidate drugs were assessed for their effect on the ISR under MG132 stress in astrocytes. The expression levels of the ISR-relevant proteins, p-eIF2 $\alpha$ (normalised to eIF2 $\alpha$ ), GADD34 and CHOP, were evaluated following candidate drug treatment (Fig. 3a-c). ISRIB markedly increased p-eIF2 $\alpha$ and decreased GADD34 and CHOP expression under MG132 stress conditions. AVex-73, berberine and deflazacort significantly decreased $\mathrm{CHOP}$ expression in the presence of MG132.

Previous cell stress and neurodegeneration studies have established ISR-modulating roles for guanabenz and ISRIB [29, 30]. Guanabenz is considered to exert cytoprotective effects by inhibiting the activity of GADD34 to recruit eIF2 phosphatases, thus prolonging translation inhibition and avoiding the added stress of resuming protein synthesis [29]. However, at concentrations of guanabenz that induced a cytoprotective effect $(5 \mu \mathrm{M})$, we did not observe a significant impact on expression of any ISR markers in astrocytes. Conversely, ISRIB-mediated cytoprotection of astrocytes $(1.25 \mu \mathrm{M})$ was associated with increased eIF2 $\alpha$ phosphorylation, and downregulation of GADD34 and CHOP.

\section{Effect of candidate drugs on indicators of mitochondrial function}

The eIF2B mutations in murine models have been shown to decrease mitochondrial membrane potential and impair mitochondrial complex I function, resulting in a compensatory increase in mitochondrial abundance [9]. Furthermore, mutations in eIF2B genes impair mitochondrial function during oxidative stress conditions in VWMD murine fibroblasts and astrocytes [11]. VWMD1 and VWMD6 patient iPSC-derived astrocytes demonstrated reduced mitochondrial membrane potential in both the presence and absence of MG132, suggesting reduced mitochondrial membrane potential is a cellular phenotype of VWMD patient cells, even under basal conditions (Figure S8).

The candidate drugs were investigated for their ability to protect against oxidative stress and improve mitochondrial membrane potential. VWMD1 EIF2B5 $5^{\mathrm{R} 113-}$ $\mathrm{H} / \mathrm{A} 403 \mathrm{~V}$ patient astrocytes treated with ursodiol or zileuton led to decreased generation of reactive oxygen species, in both the presence and absence of MG132 stress (Fig. 4a). This correlates with the purported radical scavenging activity of the 5-lipoxygenase antagonist zileuton [31]. Ursodiol also increased the relative mitochondrial membrane potential of VWMD1 EIF2B5 ${ }^{\mathrm{R} 113-}$ $\mathrm{H} / \mathrm{A} 403 \mathrm{~V}$ astrocytes under both unstressed and stressed conditions (Fig. 4b), consistent with a loss of mitochondrial membrane potential as a cellular phenotype of VWMD astrocytes. Furthermore ursodiol reduced oxidative stress in both control and VWMD astrocytes 


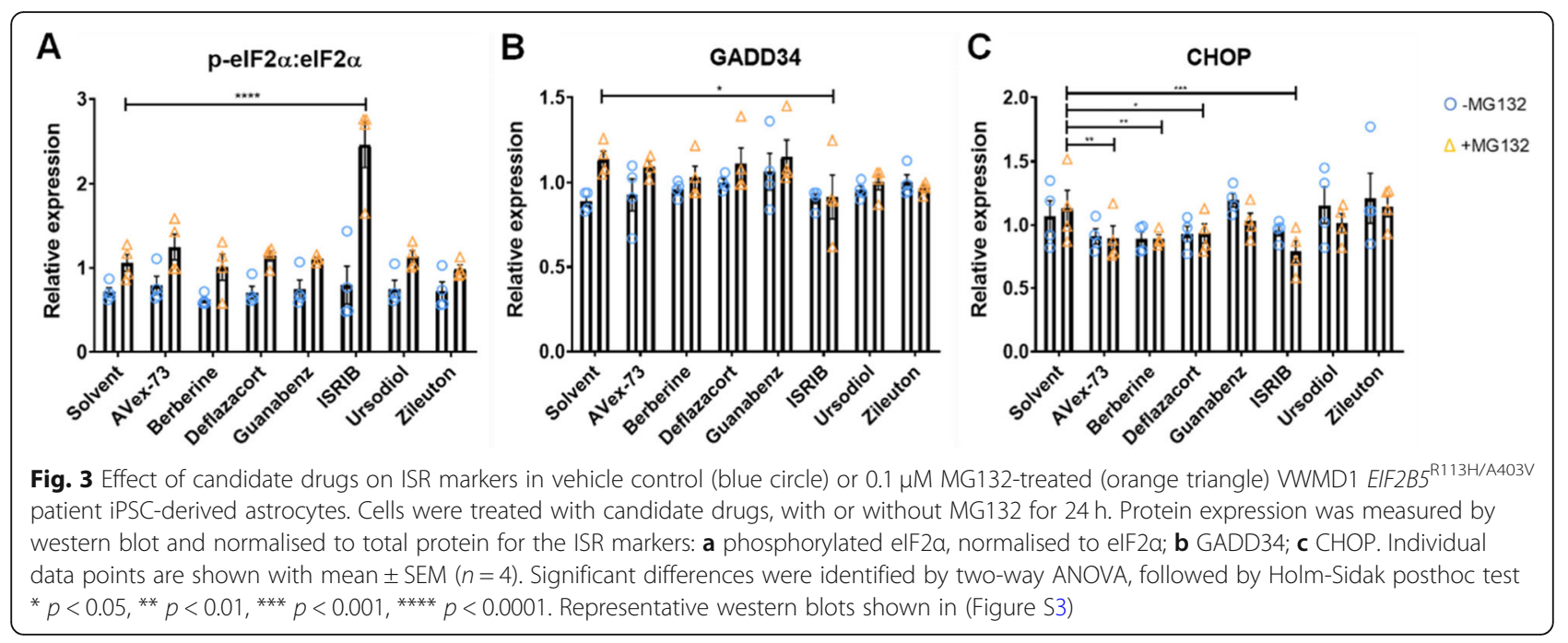

A

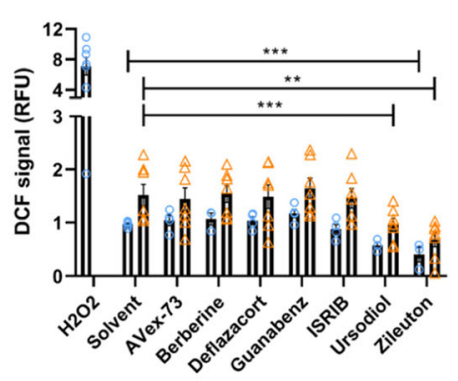

C

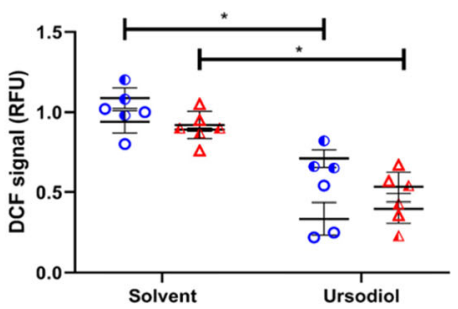

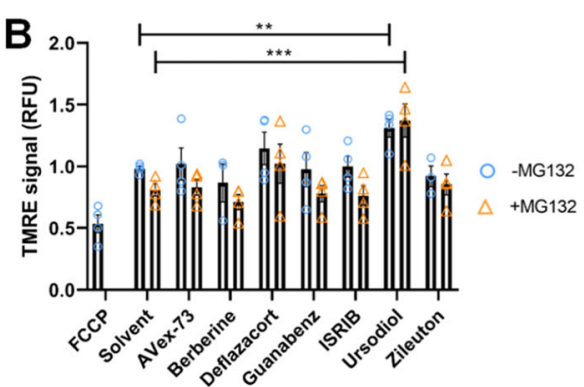

D

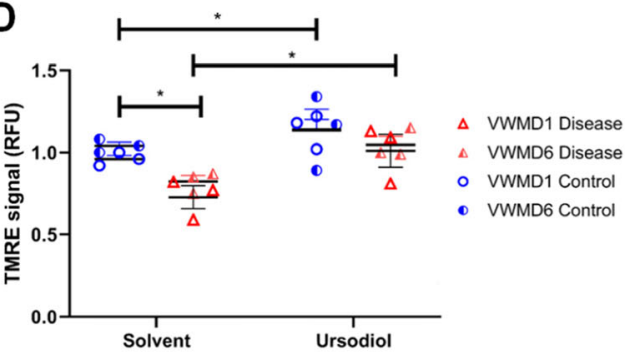

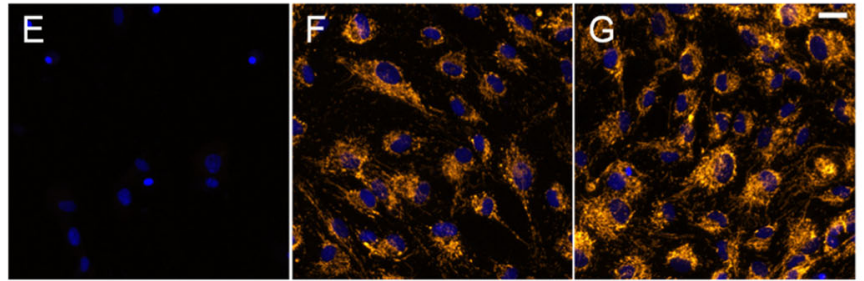

Fig. 4 Effect of candidate drugs on oxidative stress and mitochondrial membrane potential in WWMD astrocyte cell culture. WWMD1 iPSC-derived

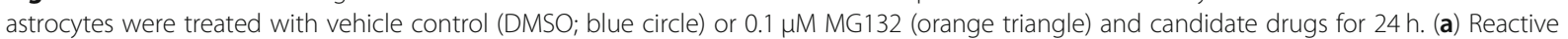
oxygen species measured via DCF fluorescence and (b) TMRE fluorescence as an indicator of mitochondrial membrane potential. Effect of ursodiol on (c) ROS generation via DCF fluorescence and (d) mitochondrial membrane potential via TMRE fluorescence in disease and control astrocyte cultures. Representative confocal fluorescence microscopy images of: (e) FCCP (protonophore, mitochondrial uncoupler, loss of TMRE fluorescence), (f) TMRE assay solvent, (g) ursodiol in WWMD1 EIF2B5 ${ }^{R 113 H / A 403 V}$ astrocytes (scale bar $=30 \mu \mathrm{m}$ ) (orange $=$ TMRE; blue $=$ Hoechst 33342 nuclear stain). For A-D individual data points represent mean \pm SEM $(n=3-6)$; significant differences were identified by two-way ANOVA followed by Holm-Sidak posthoc test ${ }^{*} p<0.05,{ }^{* *} p<0.01,{ }^{* *} p<0.001$ 
(Fig. 4c) and improved the mitochondrial membrane potential phenotype of VWMD astrocytes, bringing the levels of TMRE to those of control cells (Fig. 4d-g). Together, these data suggest that ursodiol may promote mitochondrial function and reduce oxidative stress in VWMD astrocytes.

\section{Discussion}

This study screened a panel of 2400 FDA-approved drugs, of varying classes, in an aim to identify repurposable drugs to protect against VWMD. A phenotype of VWMD cells, under basal conditions or in the presence of stress, highlighted dysfunction in the mitochondrial membrane potential of VWMD cells. Protective drugs targeted common pathways, and included compounds with anti-inflammatory activity and/or drugs purported to improve mitochondrial function or reduce oxidative stress. Steroids can regulate inflammation, mitochondrial function and apoptosis with neuroprotective effects in brain injury, Alzheimer's disease, Parkinson's disease, multiple sclerosis, and stroke [32]. Although glucocorticosteroids are a commonly administered class of drugs, an anecdotal study of corticosteroids on three VWMD patients did not identify benefits and the patients were removed from this treatment due to potential clinical complications [33]. Nonetheless, deflazacort was included in our study as a representative glucocorticosteroid on the basis of fewer reports of adverse effects in the literature and its existing use in the clinic, including in children with muscular dystrophy [34, 35]. Reduced CHOP expression in stress-induced cells following deflazacort treatment may reflect an ability of this drug to improve mitochondrial function [36].

The protective effect of the antioxidant edaravone was evident in astrocytes at a higher efficacy than any other candidate, potentially due to its well established radical scavenging activity [37]. Edaravone was recently approved for amyotrophic lateral sclerosis and while its administration is currently limited to intravenous injection, oral and mucosal formulations are in development [38-40].

Zileuton is an oral anti-asthmatic leukotriene inhibitor with reactive oxygen species scavenging activity [31]. In this study an increase in cell viability was correlated with a significant decrease in ROS generation but no significant effects on integrated stress response modulation. Its neuroprotective effects have been correlated to anti-inflammatory activity in cerebral ischemia, possibly as a result of reducing ROS-activated inflammation, and preventing glutamate oxidative toxicity via ferroptosis $[31,41]$.

ISRIB is thought to allow eIF2B to escape inhibitory complex formation with $\mathrm{p}$-eIF $2 \alpha$, thus limiting repression of protein synthesis while under ER stress [17, 26].
Treatment of EIF2B5 VWMD mice with ISRIB, or a derivative, 2BAct, ameliorated myelin loss, and improved ISR signature and motor function $[17,24,26]$. It is also worth noting that the stabilising effect of ISRIB may be dependent on the precise mutations and the cellular concentration of p-eIF $2 \alpha$, whereby it has been found to inhibit low level but not high level ISR activation [24, 42]. As ISRIB inhibits the integrated stress response, it is evident that enabling protein synthesis to resume under acute proteotoxic stress may have an adverse effect on cell survival, and the enhanced effect of cytotoxic drugs by ISRIB have been recently demonstrated in prostate and pancreatic cancer studies $[43,44]$.

The phytochemical, berberine, was included in the panel as a well-tolerated natural compound [27]. In MG132-stressed astrocytes berberine treatment increased cell viability, potentially via decreasing proapoptotic CHOP. The antioxidant, anti-inflammatory, ER and mitochondrial protective effects of berberine have been noted in numerous studies, and include increased levels of superoxide dismutase and glutathione, inhibition of apoptosis and decreased cytochrome $\mathrm{C}$ and $B A X: B C L 2$ ratio in ischemic injury and diabetic animal models [45, 46].

Overall, one of the most promising drugs identified with translational potential was ursodiol, a bile acid naturally formed in the liver and administered for gallstones. Ursodiol has a demonstrated capacity to cross the blood brain barrier, based on clinical trials for motor neurone disease, reaching levels in cerebral spinal fluid that could be protective [47]. Additionally, ursodiol has been identified as protective in other neurodegenerative and optical atrophy research, where its anti-apoptotic and neuroprotective effects were observed, although the underlying molecular mechanism of ursodiol-mediated protection was not identified [48, 49]. Although ursodiol is considered to be generally well tolerated, fatigue, nausea, weight gain, and depression are possible symptoms [50]. Progression of VWMD includes glial cell death, developing towards neuronal cell death, and leading to paralysis and neuropathy [51]. We observed ursodiol to decrease oxidative stress and increase mitochondrial membrane potential, under stressed and non-stressed conditions, and improve cell viability in VWMD astrocytes under proteasomal stress. The increase of mitochondrial membrane potential by ursodiol identified in our study is consistent with reports for Alzheimer's disease patient fibroblasts [52]. Considering disrupted mitochondrial function has been extensively implicated in VWMD and a wide range of neurodegenerative disorders, further investigation of the neuroprotective effects of ursodiol are warranted. Future studies should confirm these findings in additional VWMD patient cell lines and in vivo studies. 


\section{Conclusions}

The hypothetical premise of cytoprotective drugs for VWMD are those that can reverse cellular phenotypes and limit disease-related degeneration. Patient-derived cells are a useful resource to support drug repurposing studies for rare diseases. Together with information from previous studies on other CNS diseases [47, 52] our preliminary findings are supportive toward ongoing in vivo investigation of ursodiol, zileuton and other antiinflammatory drugs as cytoprotective agents for VWMD.

\section{Supplementary information}

Supplementary information accompanies this paper at https://doi.org/10. 1186/s41231-020-00071-0.

\section{Additional file 1}

\section{Abbreviations}

AVex-73: Anavex 2-73; CHOP: C/EBP homologous protein; CNS: Central nervous system; CNTF: Ciliary neurotrophic factor; DCF: H2DCFDA EGF: Epidermal growth factor; ER: Endoplasmic reticulum; FDA: Food and Drug Administration; FCCP: Carbonyl cyanide-4-phenylhydrazone; FGF2: Basic fibroblast growth factor; GADD34: Growth arrest and DNA damageinducible protein; iPSC: induced pluripotent stem cell; ISR: Integrated stress response; p-elF2a: phosphorylated elF2a; TMRE: Tetramethylrhodamine ethyl ester; WWMD: Vanishing white matter disease

\section{Acknowledgements}

The authors thank Dr. Gil Stynes (IHMRI, and Department of Surgery, Wollongong Hospital) for fibroblast collection and Prof Justin Yerbury, A/Prof Mirella Dottori and Prof Heath Ecroyd for valuable discussions, and Dr. Reece Gately and Rachelle Balez for technical assistance. The authors wish to thank all of the donors, the patients and their families that have made this research possible.

\begin{abstract}
Authors' contributions
NN - data generation, data analysis, manuscript writing; MC - data generation, data analysis; TB - data generation, data analysis; SM - data generation, data analysis; ME - data generation; DS - data generation; DDH data analysis; JL - data generation; SSM - data analysis; NSB - data generation; CS - data generation; LO - intellectual input, data analysis, manuscript writing, supervision, funding. All authors reviewed the manuscript. The author(s) read and approved the final manuscript.
\end{abstract}

\section{Funding}

This research was funded by philanthropic donations and a grant from the Illawarra Health and Medical Research Institute (IHMRI). L.O. is supported by a National Health and Medical Research Council (NHMRC) of Australia Boosting Dementia Research Leadership Fellowship (APP1135720).

\section{Availability of data and materials}

The authors confirm that the data supporting the findings of this study are available within the article. Data and materials may be available on request if the requests conform to the Human Research Ethics Committee approval requirements.

\section{Ethics approval and consent to participate}

All experimental protocols were approved by the University of Wollongong Human Research Ethics Committee (HE17/522). All participants provided consent to participate.

\section{Consent for publication}

Participants provided consent for publication.

\section{Competing interests}

The authors declare they have no competing interests.

\section{Author details}

${ }^{1}$ Illawarra Health and Medical Research Institute, Northfields Avenue, Wollongong, 2522 New South Wales, Australia. ${ }^{2}$ School of Chemistry and Molecular Bioscience and Molecular Horizons, University of Wollongong, Northfields Avenue, Wollongong, 2522 New South Wales, Australia. ${ }^{3}$ School of Medicine, University of Wollongong, Northfields Avenue, Wollongong, 2522 New South Wales, Australia.

Received: 18 August 2020 Accepted: 7 October 2020

Published online: 21 October 2020

\section{References}

1. Bugiani M, Vuong C, Breur M, van der Knaap MS. Vanishing white matter: a leukodystrophy due to astrocytic dysfunction. Brain Pathol. 2018;28(3):408-21.

2. Proud CG. Vanishing white matter: the next 10 years. Future Neurol. 2012; 7(1):81-92.

3. Hamilton EM, van der Lei HD, Vermeulen $G$, Gerver JA, Lourenço CM, Naidu S, et al. Natural history of vanishing white matter. J Ann Neurol. 2018;84(2): 274-88.

4. Brush MH, Shenolikar S. Control of cellular GADD34 levels by the $26 \mathrm{~S}$ proteasome. Mol Cell Biol. 2008;28(23):6989-7000.

5. Moon SL, Parker R. ElF2B2 mutations in vanishing white matter disease hypersuppress translation and delay recovery during the integrated stress response. RNA. 2018:24(6):841-52.

6. Dooves S, Bugiani M, Postma NL, Polder E, Land N, Horan ST, et al. Astrocytes are central in the pathomechanisms of vanishing white matter. J Clin Invest. 2016;126(4):1512-24.

7. Leferink PS, Dooves S, Hillen AEJ, Watanabe K, Jacobs G, Gasparotto L, et al. Astrocyte subtype vulnerability in stem cell models of vanishing white matter. Ann Neurol. 2019;86(5):780-92.

8. Zhou L, Li P, Chen N, Dai L-F, Gao K, Liu Y-N, et al. Modeling vanishing white matter disease with patient-derived induced pluripotent stem cells reveals astrocytic dysfunction. CNS Neurosci Ther. 2019;25(6):759-71.

9. Raini $G$, Sharet $R$, Herrero M, Atzmon A, Shenoy A, Geiger $T$, et al. Mutant elF2B leads to impaired mitochondrial oxidative phosphorylation in vanishing white matter disease. J Neurochem. 2017;141(5):694-707.

10. van der Voorn JP, van Kollenburg B, Bertrand G, Van Haren K, Scheper GC, Powers JM, et al. The unfolded protein response in vanishing white matter disease. J Neuropathol. 2005;64(9):770-5.

11. Atzmon A, Herrero M, Sharet-Eshed R, Gilad Y, Senderowitz H, Elroy-Stein O. Drug screening identifies sigma-1-receptor as a target for the therapy of WWM leukodystrophy. Front Mol Neurosci. 2018;11:336.

12. Herrero M, Mandelboum S, Elroy-Stein O. elF2B mutations cause mitochondrial malfunction in Oligodendrocytes. NeuroMolecular Med. 2019; 21(3):303-13.

13. Bax M, Balez R, Muñoz SS, Do-Ha D, Stevens CH, Berg T, et al. Generation and characterization of a human induced pluripotent stem cell line UOWi005-a from dermal fibroblasts derived from a CCNFS621G familial amyotrophic lateral sclerosis patient using mRNA reprogramming. Stem Cell Res. 2019:40:101530

14. Balez R, Berg T, Bax M, Muñoz SS, Cabral-da-Silva MC, Engel M, et al. The mRNA-based reprogramming of fibroblasts from a SOD1E101G familial amyotrophic lateral sclerosis patient to induced pluripotent stem cell line UOWi007. Stem Cell Res. 2020;42:101701.

15. Denham M, Dottori M. Neural differentiation of induced pluripotent stem cells. Neurodegeneration. New York: Springer; 2011. p. 99-110.

16. Vichai V, Kirtikara K. Sulforhodamine B colorimetric assay for cytotoxicity screening. Nat Protoc. 2006;1(3):1112.

17. Wong $Y L$, LeBon $L$, Basso AM, Kohlhaas KL, Nikkel AL, Robb HM, et al. elF2B activator prevents neurological defects caused by a chronic integrated stress response. ELife. 2019:8:e42940.

18. Wisse LE, ter Braak TJ, van de Beek M-C, van Berkel CGM, Wortel J, Heine VM, et al. Adult mouse elF2BE Arg191His astrocytes display a normal integrated stress response in vitro. Sci Rep. 2018;8(1):3773.

19. Park HS, Jun DY, Han CR, Woo HJ, Kim YH. Proteasome inhibitor MG132induced apoptosis via ER stress-mediated apoptotic pathway and its potentiation by protein tyrosine kinase p56lck in human Jurkat T cells. Biochem Pharmacol. 2011:82(9):1110-25.

20. Maharjan S, Oku M, Tsuda M, Hoseki J, Sakai Y. Mitochondrial impairment triggers cytosolic oxidative stress and cell death following proteasome inhibition. Sci Rep. 2014;4(1):5896. 
21. Jiang H-Y, Wek RC. Phosphorylation of the a-subunit of the eukaryotic initiation factor-2 (elF2a) reduces protein synthesis and enhances apoptosis in response to proteasome inhibition. J Biol Chem. 2005;280(14):14189-202.

22. Brush MH, Weiser DC, Shenolikar S. Growth arrest and DNA damageinducible protein GADD34 targets protein phosphatase 1 alpha to the endoplasmic reticulum and promotes dephosphorylation of the alpha subunit of eukaryotic translation initiation factor 2. Mol Cell Biol. 2003;23(4): 1292-303.

23. Teske BF, Fusakio ME, Zhou D, Shan J, McClintick JN, Kilberg MS, et al. CHOP induces activating transcription factor 5 (ATF5) to trigger apoptosis in response to perturbations in protein homeostasis. Mol Biol Cell. 2013;24(15):2477-90.

24. Abbink TEM, Wisse LE, Jaku E, Thiecke MJ, Voltolini-González D, Fritsen H, et al. Vanishing white matter: deregulated integrated stress response as therapy target. Ann Clin Transl Neurol. 2019;6(8):1407-22.

25. Dooves S, Bugiani M, Wisse LE, Abbink TEM, van der Knaap MS, Heine VM. Bergmann glia translocation: a new disease marker for vanishing white matter identifies therapeutic effects of Guanabenz treatment. Neuropathol Appl Neurobiol. 2018;44(4):391-403.

26. Wong YL, LeBon L, Edalji R, Lim HB, Sun C, Sidrauski C. The small molecule ISRIB rescues the stability and activity of vanishing white matter disease elF2B mutant complexes. Elife. 2018;7:e32733.

27. Vuddanda PR, Chakraborty S, Singh S. Berberine: a potential phytochemical with multispectrum therapeutic activities. Expert Opin Investig Drugs. 2010; 19(10):1297-307.

28. Weng T-Y, Tsai S-YA ST-P. Roles of sigma-1 receptors on mitochondrial functions relevant to neurodegenerative diseases. J Biomed Sci. 2017; 24(1):74.

29. Wang L, Popko B, Tixier E, Roos RP. Guanabenz, which enhances the unfolded protein response, ameliorates mutant SOD1-induced amyotrophic lateral sclerosis. Neurobiol Dis. 2014;71:317-24.

30. Halliday M, Radford H, Sekine Y, Moreno J, Verity N, Le Quesne J, et al. Partial restoration of protein synthesis rates by the small molecule ISRIB prevents neurodegeneration without pancreatic toxicity. Cell Death Dis. 2015;6(3):e1672-e.

31. Liu Y, Wang W, Li Y, Xiao Y, Cheng J, Jia J. The 5-lipoxygenase inhibitor zileuton confers neuroprotection against glutamate oxidative damage by inhibiting ferroptosis. Biol Pharm Bull. 2015;38(8):1234-9.

32. Garcia-Segura LM. Hormones and brain plasticity. Oxford: Oxford University press; 2009

33. Turón-Viñas E, Pineda M, Cusí V, López-Laso E, Del Pozo RL, Gutiérrez-Solana $L G$, et al. Vanishing white matter disease in a spanish population. Journal of central nervous system disease. J Cent Nerv Syst Dis. 2014;6:JCNSD. S13540.

34. Petnikota H, Madhuri V, Gangadharan S, Agarwal I, Antonisamy B. Retrospective cohort study comparing the efficacy of prednisolone and deflazacort in children with muscular dystrophy: a 6 years' experience in a south Indian teaching hospital. Indian J Orthop. 2016;50(5):551-7.

35. McAdam LC, Mayo AL, Alman BA, Biggar WD. The Canadian experience with long-term deflazacort treatment in Duchenne muscular dystrophy. Acta Myol. 2012;31(1):16-20.

36. Guiraud S, Davies KE. Pharmacological advances for treatment in Duchenne muscular dystrophy. Curr Opin Pharmacol. 2017;34:36-48.

37. Kawasaki T, Kitao T, Nakagawa K, Fujisaki H, Takegawa Y, Koda K, et al. Nitric oxide-induced apoptosis in cultured rat astrocytes: protection by edaravone, a radical scavenger. Glia. 2007;55(13):1325-33.

38. Sato T, Mizuno K, Ishii F. A novel administration route of edaravone-\|: mucosal absorption of edaravone from edaravone/hydroxypropyl-betacyclodextrin complex solution including L-cysteine and sodium hydrogen sulfite. Pharmacology. 2010;85(2):88-94.

39. Parikh A, Kathawala K, Tan CC, Garg S, Zhou X-F. Development of a novel oral delivery system of edaravone for enhancing bioavailability. Int J Pharm. 2016:515(1-2):490-500

40. Rothstein JD. Edaravone: a new drug approved for ALS. Cell. 2017;171(4):725

41. X-k T, Yang W-z, Wang C-h, S-s S, Y-I Z, C-m C, et al. Zileuton reduces inflammatory reaction and brain damage following permanent cerebral ischemia in rats. J Inflammation. 2010;33(5):344-52.

42. Rabouw HH, Langereis MA, Anand AA, Visser $L$, de Groot $R J$, Walter $P$, et al. Small molecule ISRIB suppresses the integrated stress response within a defined window of activation. Proc Natl Acad Sci. 2019;116(6):201815767.

43. Palam L, Gore J, Craven K, Wilson J, Korc M. Integrated stress response is critical for gemcitabine resistance in pancreatic ductal adenocarcinoma. Cell Death Dis. 2015;6(10):e1913-e.
44. Nguyen HG, Conn CS, Kye Y, Xue L, Forester CM, Cowan JE, et al. Development of a stress response therapy targeting aggressive prostate cancer. Sci Transl Med. 2018;10(439):eaar2036.

45. Yu W, Sheng $M, X u R, Y u J$, Cui $K$, Tong J, et al. Berberine protects human renal proximal tubular cells from hypoxia/reoxygenation injury via inhibiting endoplasmic reticulum and mitochondrial stress pathways. J Transl Med. 2013;11(1):24.

46. Li Z, Geng Y-N, Jiang J-D, Kong W-J. Antioxidant and anti-inflammatory activities of berberine in the treatment of diabetes mellitus. Evid Based Complement Altern Med. 2014;2014:289264.

47. Parry GJ, Rodrigues CM, Aranha MM, Hilbert SJ, Davey C, Kelkar P, et al. Safety, tolerability, and cerebrospinal fluid penetration of ursodeoxycholic acid in patients with amyotrophic lateral sclerosis. J Clin Neuropharmacol. 2010;33(1):17-21.

48. Boatright $\mathrm{JH}$, Nickerson JM, Moring AG, Pardue MT. Bile acids in treatment of ocular disease. J Ocul Biol. 2009;2(3):149.

49. Foster SL, Kendall C, Lindsay AK, Ziesel AC, Allen RS, Mosley SS, et al. Development of Bile Acids as Anti-Apoptotic and Neuroprotective Agents in Treatment of Ocular Disease. Drug Product Dev for the Back of the Eye. Boston: Springer; 2011. p. 565-76

50. National Institute of Health. LiverTox: Clinical and Research Information on Drug-Induced Liver Injury. Ursodiol (Ursodeoxycholic Acid). 2012. Available from: https://www.ncbi.nlm.nih.gov/books/NBK548309/. Accessed 15 Sept 2020

51. Barros SR, Parreira SC, Miranda AF, Pereira AM, Campos NM. New insights in vanishing white matter disease: isolated bilateral optic neuropathy in adult onset disease. J Neuroophthalmol. 2018;38(1):42-6.

52. Bell SM, Barnes K, Clemmens H, Al-Rafiah AR, Al-ofi EA, Leech V, et al. Ursodeoxycholic acid improves mitochondrial function and redistributes Drp1 in fibroblasts from patients with either sporadic or familial Alzheimer's disease. J Mol Biol. 2018:430(21):3942-53.

\section{Publisher's Note}

Springer Nature remains neutral with regard to jurisdictional claims in published maps and institutional affiliations.

Ready to submit your research? Choose BMC and benefit from

- fast, convenient online submission

- thorough peer review by experienced researchers in your field

- rapid publication on acceptance

- support for research data, including large and complex data types

- gold Open Access which fosters wider collaboration and increased citations

- maximum visibility for your research: over $100 \mathrm{M}$ website views per year

At $\mathrm{BMC}$, research is always in progress.

Learn more biomedcentral.com/submissions 